\title{
Transgenic expression of Notch-1 intracellular active domain (N1IC) in T cells: a potential therapy to overcome suppression induced by tumors
}

\author{
Rosa A Sierra, Dorota Wyczechowska, Paulo C Rodriguez \\ From Society for Immunotherapy of Cancer 28th Annual Meeting \\ National Harbor, MD, USA. 8-10 November 2013
}

An impaired anti-tumor immunity is found in patients with cancer and represents a major obstacle in the successful development of different forms of immunotherapy. Several pathways have been described to explain the induction of $\mathrm{T}$ cell anergy in tumors; however, the translation of this understanding into new adjuvants for immunotherapy has been limited. Pharmacological inhibition of Notch-homolog-related proteins (Notch) in T cells leads to a similar inhibitory effect to that found in tumors. Thus, we aimed to determine the role of Notch in the function of $\mathrm{T}$ cells and to test the effect of the transgenic expression of Notch 1 intracellular active domain (N1IC) in a tumor model of $\mathrm{T}$ cell-based immunotherapy. Our results show an increased expression of Notch 1 and 2, but not Notch 3 and 4, in activated CD4+ and CD8+ $\mathrm{T}$ cells. In addition, conditional deletion of both Notch 1 and 2 in antigen-specific CD4+ and CD8+ T cells, but not individual deletion of Notch forms, prevented cell proliferation and IFN $\gamma$ production. Interestingly, tumor-associated myeloid-derived suppressor cells (MDSC) inhibited the expression of Notch $1 / 2$ in activated $T$ cells at the same ratios at which they blocked $\mathrm{T}$ cell proliferation and IFN $\gamma$ production. To address the relevance of the decreased expression of Notch in T cells in tumor-induced tolerance, we adoptively transferred antigen-specific CD8+ $\mathrm{T}$ cells over-expressing N1IC (OT-1 N1ICf/f Gnz-Cre+/-) or floxed controls, into mice bearing established $3 \mathrm{LL}$ tumors carrying the model antigen ovalbumin (OVA). A higher anti-tumor effect and IFN $\gamma$ production was observed in tumor-bearing mice transferred with N1IC CD8 + cells, as compared to those receiving floxed cells. Then, we tested whether N1IC directly promoted $\mathrm{T}$ cell cytotoxicity. An increased antigen-specific killing against OVA-loaded cells was induced by N1IC CD8+ cells in vitro and in vivo, as compared to control cells. This effect correlated with an increased expression in N1IC CD8+ $\mathrm{T}$ cells of the degranulation marker CD107a and the effector molecules granzyme B and IFN $\gamma$. Interestingly, N1IC endogenously bound to granzyme $B$ promoter, suggesting a direct role of N1IC in the induction of effector genes in $\mathrm{T}$ cells. Moreover, an increased expression of $\mathrm{T}$ cell survival receptors CD122, IL-7R, and CD44 was found in N1IC mice, suggesting a potential role of Notch signaling in $\mathrm{T}$ cell survival/stemness. Altogether, these results suggest the role of Notch-1/2 in $\mathrm{T}$ cell function and the potential use of N1IC as an adjuvant for T cell-based immunotherapy. Continuation of this work could enable the design of new therapeutic approaches to reverse $\mathrm{T}$ cell anergy in individuals with cancer.

Published: 7 November 2013 